\title{
Media Interview Skills ${ }^{1}$
}

\author{
Ricky Telg²
}

This publication about media interview skills is the fourth of a four-part series on media relations. This series also covers media relations strategies, news releases and public service announcements, and working with the media.

\section{Introduction}

Being interviewed by the news media is another component of an effective media relations strategy. Many people assume that being interviewed is as simple as walking into an office or television studio and waiting for a reporter to ask questions. However, if you are not fully prepared, both in terms of what you want to say and what you expect during an interview, being interviewed may be a frightening experience. On the other hand, if you know what you want to say and have confidence in your ability and appearance, an interview can be a rewarding and enjoyable experience. This publication focuses on basic media interview skills to help you feel comfortable during an interview.

\section{Preparing}

When a reporter calls, you should find out his or her name and employer, what the story is about, what the deadline is, and what is expected of you. If you cannot answer the reporter's questions, try to direct the reporter to someone who can.

If you are the right person to answer the reporter's questions, you need to know how best to present yourself in an interview. The following recommendations can help you succeed in most interviews. The interview skills described in this section pertain to all forms of media unless otherwise noted.

Prepare two to three ideas you want to convey. These are your communication points, also known as "talking points." Your communication points are the most important issues or points you hope to address with the reporter during the interview. Anticipate issues and questions that may arise during the interview and be prepared to use those issues to launch your communication points. To help you develop your communication points, write three sentences that you could use to convey each point. If they are long sentences, keep refining. Develop at least one answer you can give in 20 seconds or less. Then practice saying your communication point aloud. You also may wish to prepare three questions you dread for the reporter to ask. Develop responses to each question. The questions in the interview probably will not be as bad as you have imagined, but it is best to be prepared.

Make short, simple, specific statements. As you prepare your communication points, practice using simple, everyday language and short answers. Try to keep answers for recorded television or radio interviews to 20 seconds or less. Use real-life examples. Explain your most important point first. Also, summarize and then elaborate, such as in this example: "We have the best organization in the area because our members really care. Let me explain what I mean...."

1. This document is WC114, one of a series of the Department of Agricultural Education and Communication, Florida Cooperative Extension Service, Institute of Food and Agricultural Sciences, University of Florida. Original publication date August 2011. Visit the EDIS website at http://edis.ifas.ufl. edu.

2. Ricky Telg, professor, Department of Agricultural Education and Communication, Florida Cooperative Extension Service, Institute of Food and Agricultural Sciences, University of Florida 
Pause after complete statements. The radio or television reporter will appreciate these breaks during the editing process.

Do not ramble. When you think you have answered a question adequately, do not feel compelled to keep talking simply because the interviewer has a microphone up to your mouth. If you are satisfied with your answer, stop talking. Rambling can lead you to say the wrong thing.

Do not refer to the reporter or to previous answers. In a radio or television interview, do not say the reporter's name in the middle of a sentence. Also, do not use the phrase, "As I explained earlier." They are difficult to edit out. Here is an example: "We have the best organization in the area, as I explained earlier, because our members really care." If the phrase cannot be edited out, viewers may not know what you and the reporter have discussed previously and may not understand to what you are referring.

Think before you speak. Avoid verbal fillers, such as "uh," "ah," "well," "yeah," and "you know," especially for radio and television interviews.

Maintain eye contact with the reporter. If you are doing a television interview, do not look at the camera; look the interviewer in the eye the entire time. Maintaining eye contact gives you credibility and implies you believe what you are saying.

\section{Overcoming Voice Problems}

For recorded interviews (radio or television), the impact of your spoken message may depend on how you speak. The sound of your voice determines how well you hold the attention of the audience who listens to you. The ability to speak well can be improved through practice. Common voice problems involve pitch, rate, and articulation.

Pitch is the high and low sounds of your voice. The habit of inflecting up (raising the sound) at the ends of sentences and phrases is a pitch problem because it sounds like you are always asking a question. Making everything you say sound like a question undermines your authority. You will sound more assertive if you lower your pitch and inflect downward.

The speed at which you talk is your speaking rate. While sprinting through your message may leave listeners behind, talking too slowly may bore them. To find out if you need to slow down or speed up, record yourself talking with someone, preferably in a mock news interview situation.
Play the recording and listen to how quickly or slowly you speak. Practice establishing a rate that is easy for people to understand. Once you have established a good pitch and rate, practice varying them, along with your volume, to add emphasis and expression to your message.

Lastly, consider how you articulate - or distinctly pronounce-words. Do not run your words together. Proper articulation helps your listening audience understand your words.

\section{Mock News Interviews}

You can practice before an actual interview by conducting a mock news interview. A mock news interview is when someone acts like a reporter and asks you questions that a "real" reporter would ask. You may wish to record the mock interview so you can review and critique your performance. Following are some suggestions on how to answer questions or prepare for real or mock news interviews so that you do not succumb to stage fright:

Concentrate on the question you are being asked. Pause before answering a question just long enough to formulate an outline of the answer.

Before the interview starts, take a deep breath, take a drink of water, laugh, or yawn. Why should you yawn? You cannot yawn and be tense at the same time. Even a nervous laugh to yourself before the interview helps relieve tension. Any of the practices in this recommendation will help you relax.

Remind yourself that you were asked to be interviewed because you know the topic. You are the expert.

Prior to the interview, review previously recorded interviews of yourself to identify presentation strengths and weaknesses.

Try to convince yourself you are having a normal, everyday conversation with someone.

Do not eat certain foods or drink certain beverages before an interview. Certain foods and beverages coat your throat, causing difficulty in swallowing and speaking. Before the interview, stay away from such items as cola drinks, chocolates, and dairy products. It takes several hours to "uncoat" your throat from these foods and beverages.

Following are some suggestions on how to conduct a mock news interview and how to ask questions like a reporter:

Use open-ended questions. Questions that begin with "who," "what," "where," "when," "why," and "how" are best. 
Avoid questions that would have "yes" and "no" answers. Avoid questions that begin with "do you," "have you," or "were you." These are questions that provide only "yes" and "no" answers.

Avoid compound questions. A compound question asks two or more questions in one. Only ask one question at a time. This is an example of a compound question: "What are your opinions regarding this proposed change, and if you are in favor of it, why? Or if you're opposed to it, what should be done?"

Be a good listener. Show an interest in the person being interviewed.

Maintain good eye contact with the person being interviewed.

Avoid saying "uh-huh" and "I see" while the person is talking. Acknowledge responses by silently nodding or smiling.

\section{Making a Good Appearance}

Television viewers judge the trustworthiness of your message both on what you say and on your appearance. Following are a few suggestions for dressing for success in a television interview:

Clothing in a television studio setting: If you are not in the official attire of your organization, stick to a conservative, professional appearance. Avoid tight stripes or plaids. On camera, they sometimes cause the colors to "vibrate." This is called a moire (more-AY) effect. Wear midtone colors. Extremely dark or bright clothes can make your face look washed out or dark under television studio lighting. In both television studio interviews and on-location interviews, your blouse or shirt should have a place to clip a microphone.

Clothing in an on-location setting: If you are not in the official attire of your organization, your clothing should be consistent with your surroundings. For example, you are not expected to wear a suit if you are being interviewed in a peanut field or a citrus grove. Instead, wear jeans and a regular shirt. Avoid hats, but if you must wear one, push back the brim so people can see your eyes.

Jewelry: Wear only a few pieces of jewelry. Avoid "clunky" or dangling necklaces or earrings because big gold or highgloss jewelry can reflect studio lights. Short necklaces are best; long necklaces can rub against clip-on microphones.

Makeup: A woman's everyday makeup should be fine. Use a matte finish to reduce shine. Use a lipstick that is not shiny.
Men, most likely, will not have to wear makeup but should be open to the suggestion. The lighting at some television studios may cause men to look washed out; therefore, men may need makeup to highlight their facial features. If any makeup is needed for a man, television producers will apply it.

Enthusiasm: Be animated. Use gestures, facial expressions, and body language to add vitality to your words. However, be careful not to overdo it. Smile-a good first impression can help establish your credibility. Be conversational and deliver your message with confidence.

Body language: Look at the interviewer, not the video camera. Sit still in your chair because rocking or swiveling can take you out of a camera shot.

Gum and pocket change: Do not chew gum or play with your pocket change or keys while on the air.

Light-sensitive eyeglasses: Do not wear light-sensitive eyeglasses. Studio lighting and sunlight make your glasses darker, and viewers will not be able to see your eyes.

\section{Interview Checklist}

In addition to the previous suggestions, you may wish to use the following checklist to make sure you have everything covered before the media interview:

Are you familiar with the television program or publication that is interviewing you? If not, it probably would be a good idea to watch a program or two or read the publication before the interview so that you can answer questions that would appeal to their audience.

Are you the only person being interviewed? Will many others be interviewed?

Will this interview be live or taped? Will there be call-in questions?

Can you explain your communication points in a concise manner?

Have you developed a conversational style?

Have you rehearsed all possible questions and answers with someone else?

Are you aware of your body language and facial expressions? Are you ready to present your message without using jargon? 


\section{After the Interview}

After the interview, you should evaluate how well you did before you do another interview. Following are some questions you may wish to ask yourself to evaluate your interview skills:

Did you cover your communication points?

Did you create sound bites (short quotations) that were easily understandable?

Did you listen carefully to the interviewer's questions?

Did you use short sentences?

Did you maintain good eye contact with the interviewer?

Were you aware of your body language?

Did you project a strong, positive image of a person people would trust?

\section{"Be Attitudes"}

By following these final "be attitudes,"you should be successful in any interview setting:

Be prepared. Prepare in advance two or three communication points you wish to convey. Try to anticipate questions you could be asked.

Be positive. End every answer on a positive, upbeat note.

Be honest. Always tell the truth. Your credibility is crucial.

Be brief. Crystallize your ideas into a few short phrases that summarize what you are trying to communicate.

Be yourself. Keep your voice at an even pace. Act naturally.

Be energetic. Be animated. Use gestures, facial expressions, and body language to add vitality to your words. Be careful not to overdo it.

Be focused. Give the interviewer your full attention. Look squarely at the person asking the questions. Do not be concerned with distractions.

Be comfortable, confident, and take charge. Relax. You know more about the topic than the interviewer. If not, you would not be interviewed.

\section{Additional Information}

Diggs-Brown, B., \& Glou, J. (2004). The PR style guide: Formats for public relations practice. Belmont, CA: Wadsworth.

Marsh, C., Guth, D. W., \& Short, B. P. (2005). Strategic writing: Multimedia writing for public relations, advertising, sales and marketing, and business communication. Boston, MA: Pearson Education.

Telg, R. (2000). Getting out the news. Retrieved October 23, 2010, from http://mediarelations.ifas.ufl. edu/2effectivemediarelations.htm 